\title{
Article
}

\section{Simultaneous dynamic electrical and structural measurements of functional materials}

\author{
Serban, Lepadatu
}

Available at https://clok.uclan.ac.uk/12681/

Serban, Lepadatu orcid iconORCID: 0000-0001-6221-9727 (2015)

Simultaneous dynamic electrical and structural measurements of functional materials. Review of Scientific Instruments, 86 . p. 103901. ISSN 0034-6748

It is advisable to refer to the publisher's version if you intend to cite from the work. http://dx.doi.org/10.1063/1.4931992

For more information about UCLan's research in this area go to http://www.uclan.ac.uk/researchgroups/ and search for < name of research Group>.

For information about Research generally at UCLan please go to http://www.uclan.ac.uk/research/

All outputs in CLoK are protected by Intellectual Property Rights law, including Copyright law. Copyright, IPR and Moral Rights for the works on this site are retained by the individual authors and/or other copyright owners. Terms and conditions for use of this material are defined in the policies page.

\section{CLoK}

Central Lancashire online Knowledge www.clok.uclan.ac.uk

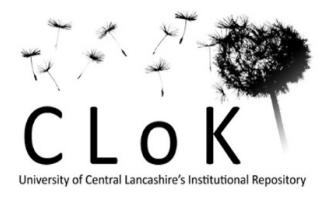




\section{A|P| $\mid \begin{aligned} & \text { Review of } \\ & \text { Scientific Instruments }\end{aligned}$}

\section{Simultaneous dynamic electrical and structural measurements of functional materials}

C. Vecchini, P. Thompson, M. Stewart, A. Muñiz-Piniella, S. R. C. McMitchell, J. Wooldridge, S. Lepadatu, L. Bouchenoire, S. Brown, D. Wermeille, O. Bikondoa, C. A. Lucas, T. P. A. Hase, M. Lesourd, D. Dontsov, and M. G. Cain

Citation: Review of Scientific Instruments 86, 103901 (2015); doi: 10.1063/1.4931992

View online: http://dx.doi.org/10.1063/1.4931992

View Table of Contents: http://scitation.aip.org/content/aip/journal/rsi/86/10?ver=pdfcov

Published by the AIP Publishing

\section{Articles you may be interested in}

Modeling of resonant magneto-electric effect in a magnetostrictive and piezoelectric laminate composite structure coupled by a bonding material

J. Appl. Phys. 112, 064109 (2012); 10.1063/1.4752271

Effects of electrostatic force on piezoelectric materials under high electric field: Impact on GaN-based nanoscale structures

J. Appl. Phys. 109, 013513 (2011); 10.1063/1.3524259

Structural And Electrical Analysis Of Lead Free BZT-xBCT Ceramics

AIP Conf. Proc. 1313, 269 (2010); 10.1063/1.3530514

Scaling of structure and electrical properties in ultrathin epitaxial ferroelectric heterostructures

J. Appl. Phys. 100, 051609 (2006); 10.1063/1.2337363

Piezoelectric characterization and thermal stability of a high-performance $\alpha$-quartz-type material, gallium arsenate

J. Appl. Phys. 97, 074110 (2005); 10.1063/1.1874293

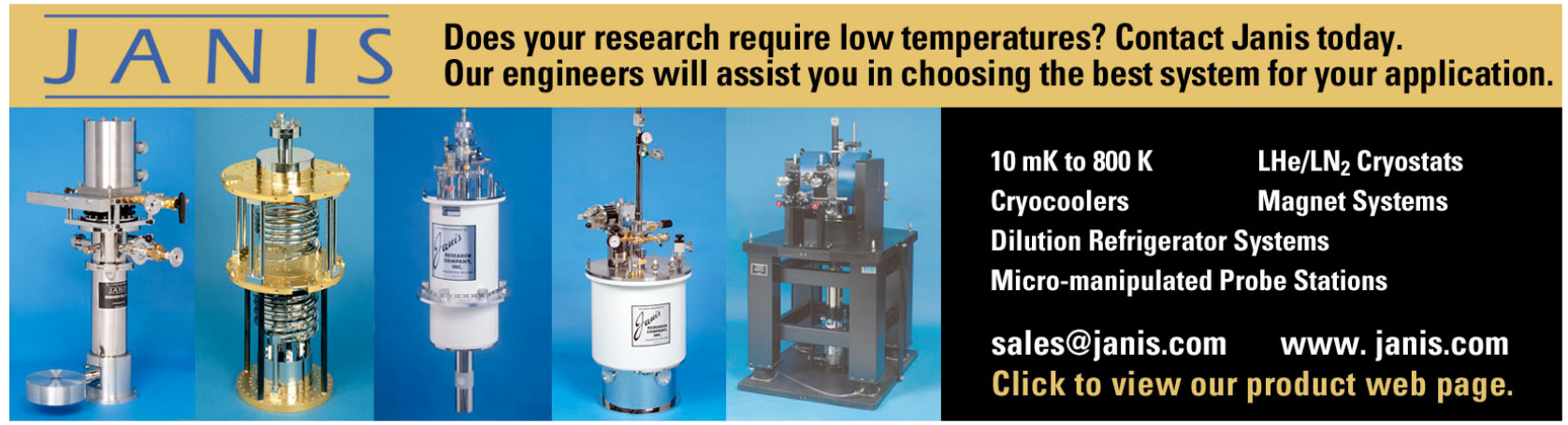




\title{
Simultaneous dynamic electrical and structural measurements of functional materials
}

\author{
C. Vecchini, ${ }^{1}$ P. Thompson, ${ }^{2,3}$ M. Stewart, ${ }^{1}$ A. Muñiz-Piniella, ${ }^{1}$ S. R. C. McMitchell,,${ }^{2,3}$ \\ J. Wooldridge, ${ }^{1}$ S. Lepadatu,,$^{1,4}$ L. Bouchenoire, ${ }^{2,3}$ S. Brown, ${ }^{2,3}$ D. Wermeille, ${ }^{2,3}$ \\ O. Bikondoa, ${ }^{2,5}$ C. A. Lucas, ${ }^{2,3}$ T. P. A. Hase,${ }^{2,5}$ M. Lesourd, ${ }^{6}$ D. Dontsov, ${ }^{7}$ and M. G. Cain ${ }^{1,8}$ \\ ${ }^{1}$ National Physical Laboratory, Hampton Road, Teddington TW11 OLW, United Kingdom \\ ${ }^{2} X M a S$, The UK-CRG, ESRF-The European Synchrotron, CS40220, F-38043, Grenoble Cedex 09, France \\ ${ }^{3}$ Department of Physics, University of Liverpool, Liverpool L69 3BX, United Kingdom \\ ${ }^{4}$ Jeremiah Horrocks Institute, University of Central Lancashire, Preston PR1 2HE, United Kingdom \\ ${ }^{5}$ Department of Physics, University of Warwick, Coventry CV4 7AL, United Kingdom \\ ${ }^{6}$ ESRF-The European Synchrotron, CS40220, F-38043, Grenoble Cedex 09, France \\ ${ }^{7}$ SIOS Meßtechnik GmbH, Am Vogelherd 46, 98693 Ilmenau, Germany \\ ${ }^{8}$ Electrosciences Ltd., Farnham, Surrey GU9 9QT, United Kingdom
}

(Received 24 July 2015; accepted 17 September 2015; published online 5 October 2015)

A new materials characterization system developed at the XMaS beamline, located at the European Synchrotron Radiation Facility in France, is presented. We show that this new capability allows to measure the atomic structural evolution (crystallography) of piezoelectric materials whilst simultaneously measuring the overall strain characteristics and electrical response to dynamically (ac) applied external stimuli. (C 2015 AIP Publishing LLC. [http://dx.doi.org/10.1063/1.4931992]

\section{INTRODUCTION}

The physical properties and functional efficiency in many real systems are intimately related to their internal stresses and strains. The optimization and maximization of material functional properties under external stimuli are continuously sought. Recently, there has been considerable research efforts in understanding the complex interplay between sample structure and internal strain which results in ferroic order. In ferroelectric materials, the direction of the internal electrical polarization $(\mathrm{P})$ can be switched by the application of an external electric field (E). The usual method for identifying and characterizing such materials is to map the hysteretic relationship between these two parameters, known as a PE loop. ${ }^{1}$ These materials often exhibit phase transformations dependent on multiple parameters such as chemical composition, temperature, electric field, stress, and history of electric field poling. ${ }^{2-5}$ In piezoelectrics, the change in polarization is associated with a mechanical change in shape and vice versa.

The strain originates from different contributions: intrinsic effects such as electronic $\left(\epsilon_{\text {elec }}\right)$ and ionic polarization $\left(\epsilon_{i o n}\right)$ and extrinsic effects such as orientation $\left(\epsilon_{o r}\right)$, space charge $\left(\epsilon_{s c}\right)$, and domain wall $\left(\epsilon_{d w}\right)$ polarizations. ${ }^{6-11}$ Each contribution stems from a short range movement of charges in response to an electric field on different time scales and hence in different frequency regimes. Intrinsic effects, related to lattice parameters changes, can be probed by diffraction ${ }^{12}$ while extrinsic contributions such as domain dynamics can be measured via interferometry. ${ }^{13}$ In order to rationalize the properties of ferroelectrics, both intrinsic and extrinsic contributions need to be assessed. While it is possible to independently measure each contribution, due to the complexity and the large parameter space exhibited by these materials, ${ }^{14-16}$ it is at the same time very challenging but crucial to perform combined and simultaneous measurements.
Novel patterned architectures utilizing ferroelectric materials have recently been proposed as a possible alternative route to replace current CMOS technology thanks to their larger operation speed and significantly lower power consumption. ${ }^{17}$ In order to support the exploitation of this transformative technology and since most of the devices operate and are controlled through application of an electric field, it is crucial to investigate the physical deformation and strain state of these inherently non-linear hysteretic materials occurring under applied electric fields, that is, in situ and in operando. The strain properties at device level can be connected to the atomic origins of strain by combining interferometric global strain measurements and x-ray diffraction (XRD). Moreover, any experimental setup requires dynamical data collection to probe the material properties in the same (or similar) conditions to those used in real industrial/technological applications (e.g., cyclical loading at various frequencies). The requirement to understand nanoscale strain behavior in this manner is the driving force behind the development of an in situ interferometric measurement system on the XMaS beamline ${ }^{18,19}$ at the European Synchrotron Radiation Facility (ESRF) in Grenoble, France.

The key to developing a useful experimental setup is to synchronize accurately the timing between the diffraction, electric polarization, and interferometry data collection as a function of the applied electric field. This paper describes how this difficult task was tackled. We also illustrate the method by showing the correlation between intrinsic strain and interferometrically measured macroscopic strain for single crystal piezoelectric materials in relation to the key parameter of interest for device applications. Experimental procedures have been developed in order to minimize measurement noise and to allow future extensions of the presented system for temperature and magnetic field control. The newly developed metrology technique represents an extremely valuable tool 
facilitating the design, optimization, and control of new piezoelectric based devices. In addition, the possibility to apply in situ electric fields and to measure displacements from picometres to micrometres enables new physics of complex materials to be explored by in operando measurements in application areas including advanced materials for energy (fuel cells, PVs, and electrochemistry) and novel sensors and actuators (magnetostriction, multiferroics, and magnetoelectrics), for example.

\section{EXPERIMENTAL TECHNIQUES}

In order to use $\mathrm{X}$-ray diffraction to investigate the dynamic switching process in ferroelectric materials, time-resolved data need to be captured. This capability has been developed at the XMaS beamline, ESRF, France by the NPL and XMaS groups. $^{20}$

\section{A. Interferometry}

The calculation of the indirect piezoelectric coefficients necessitates the measurements of small displacements and applied electrical fields. The highest recorded piezoelectric coefficient of currently known (single crystal) materials is typically in excess of $2000 \mathrm{pm} / \mathrm{V}$, but many practical systems (including constrained thin films and ceramic Lead Zirconate Titanate (PZT)) have far smaller piezoelectric coefficients. Also, with the continuing trend towards miniaturization and particularly with the introduction of Micro-ElectroMechanical systems (MEMS), there is a requirement to measure piezoelectric coefficients on physically smaller structures. Additionally, the measurement method needs to be contactless in order to minimize any mechanical interference between the measurement probe and the sample. Laser interferometer based systems are the ideal tool to satisfy this measurement need and, coupled with the knowledge of the illumination wavelength, ultimately provide a traceable measurement.

A synchrotron beamline, where a sample is mounted on a large diffractometer that is used to align and move the sample in angular space to meet the diffraction condition for different reflections, represents a very challenging environment for interferometry measurements. Interferometric measurements are traditionally performed in a laboratory environment, where the equipment is usually isolated from external vibrations and electrical noise. On the beamline, these vibrations were characterized by measuring $x, y$, and $z$ accelerations at different positions on the Huber diffractometer under static and operational conditions, as detailed below. Since a beamline has hundreds of motors/pumps and other possible sources of vibrations, the first step in minimizing the background vibration is to use a dual beam interferometer. One of the two beams will measure the sample displacement while the other will act as a reference (Fig. 1). The interferometer needs to be rigidly attached to the sample and, at the same time, allows for the X-rays to illuminate the specimen. In the present case, the interferometer has been provided by SIOS Meßtechnik GmbH. ${ }^{21}$ Developments have also been carried out to enable reliable displacement measurements in this challenging environment. These changes are described below and schematically shown in Fig. 2.

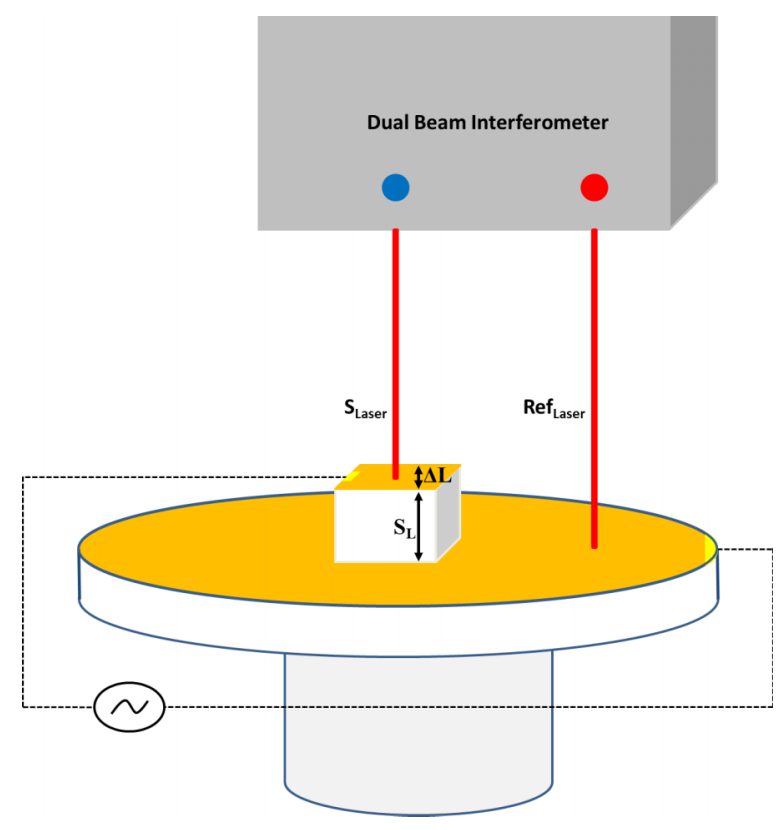

FIG. 1. Schematic of the sample setup at the XMaS beamline, ESRF. The dual beam interferometer is aligned to the sample and the reference surface, respectively. The measured displacement is $\left(\Delta \mathrm{L}_{\text {Sample }}=\Delta \mathrm{L}-\Delta \mathrm{L}_{\text {reference }}\right.$ (vibrations)).

\section{B. Michelson interferometers}

The homodyne interferometers used in this work are Michelson interferometers. Both the laser light source and power-supply/signal processing unit are separated from the measurement head. Light from the frequency-stabilized $\mathrm{HeNe}$ laser is transmitted to the measurement head on a single-mode fiber-optic lightguide, keeping heat sources well away from the measurement locations. The advantage of the metrological method presented here is based on transmitting just a single beam that is retroreflected by the moving mirror around the measurement axis. This allows configuration of the metrological setup such that there is a well-defined point of contact

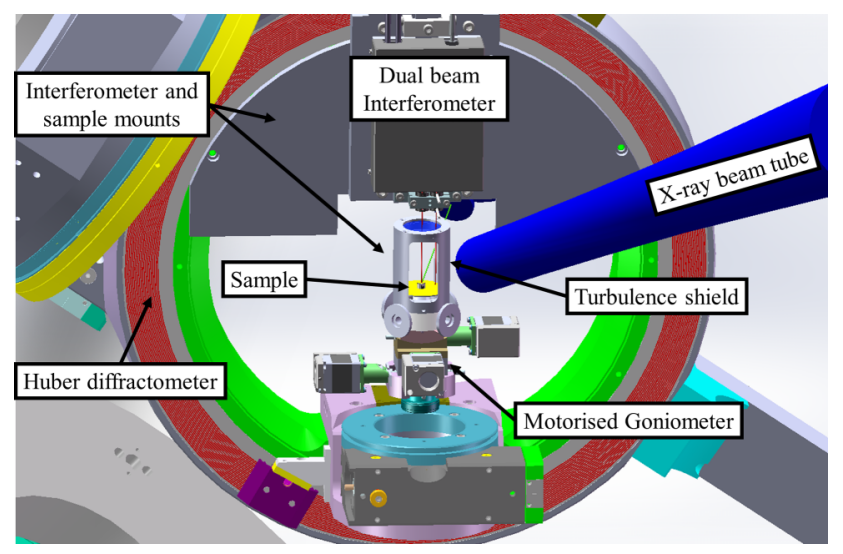

FIG. 2. Schematic of the experimental setup at the XMaS beamline, ESRF. The dual beam interferometer is rigidly connected to the $\chi$ circle of the Huber diffractometer. One laser beam is aligned with the centre of rotation of the diffractometer. The sample is mounted inside a turbulence shield. Kapton windows and an optical window seal the sample enclosure to allow $\mathrm{X}$-rays and lasers transmission, respectively. The sample is mounted on a low vacuum chamber with feedthroughs for electrical connections. 
with the object being measured. In this way, the laser beam remains aligned accurately on the measurement axis, which, in turn, means that the configuration of an Abbe comparator is maintained. The double-beam miniature interferometer has a pair of independent interferometer channels and a length resolution of $<0.1 \mathrm{~nm}$ over an angular resolution of 0.001 arc sec or better, for a baseline beam separation of $25.4 \mathrm{~mm}$. Employment of a pair of parallel measurement beams allows two pathlengths to be acquired simultaneously. For the presented application, SIOS Meßtechnik GmbH's interferometers have been improved by increasing the internal symmetry. As a result, the increased parallelism of the output beams reduces the metrological errors of the differential measurement, achieving resolutions of $\leq 0.1 \mathrm{~nm}$.

\section{System integration}

The XMaS beamline is equipped with an eleven-axis $\mathrm{Hu}-$ ber diffractometer, with the sample mounted on a " $\phi$ " rotation on top of a " $\chi$ " circle. The interferometer was mounted, through an aluminum plate adapter, directly on the $\chi$ circle, ensuring a rigid sample-interferometer system (Fig. 2). A sample holder was designed, fabricated, and mounted on a small Huber motorized goniometer, attached on the $\phi$ motor. The sample holder was designed to allow high voltage cables to be connected to the sample. In addition, a Kapton (DuPont ${ }^{\mathrm{TM}}$ ) tent was constructed to minimize air turbulence in the interferometer's optical path and therefore reduce the background noise as well as increase the measured resolution of the system. Additionally, this tent creates a low environmental vacuum to minimize air scattering (X-rays), to limit moisture on the specimen. In this case, the tent has been used to drive nitrogen across the sample therefore mitigating effects of ozone on the dielectric breakdown. ${ }^{22,23}$ The safety enclosure also protects the user against high voltage (up to $10000 \mathrm{~V}$ ). The interferometer has been mounted with the "sample" laser passing through the center of rotation of the diffractometer. The laser can be adjusted with (manual) tip and tilt controls. Also, in order to take into account alignment issues (e.g., misalignment between sample and mirror surfaces), wedge focusing lenses have been mounted in front of each laser to allow fine tuning of the laser position and direction, with an acceptance angle up to $1^{\circ}\left( \pm 0.5^{\circ}\right.$ with respect to the straight reflection).

\section{Electronics}

The analogue signal from the laser electronic box is connected directly to the ESRF designed "MUSST" (Multipurpose Unit for Synchronization, Sequencing, and Triggering) card (Fig. 3). The existing PE measurements setup was described in more detail in Ref. 20. The $\mathrm{x}$ ray point detector (avalanche photodiode-APD), the x-ray monitor (ion chamber) as well as the waveform generated by the function generator, and the measured electrical signal from the specimen (either charge or current, depending on the setup) are also connected directly to the card. The analogue signals from the two laser interferometers are also connected to the acquisition card. Finally, the synchronization signal from the function generator is connected to the trigger input of the MUSST card. The diffraction data (XRD) are recorded as a function of applied electric field, simultaneously with the APD at a single point in reciprocal space.

The XRD data are recorded in real time, synchronized with the polarization current/charge data-stream and the data

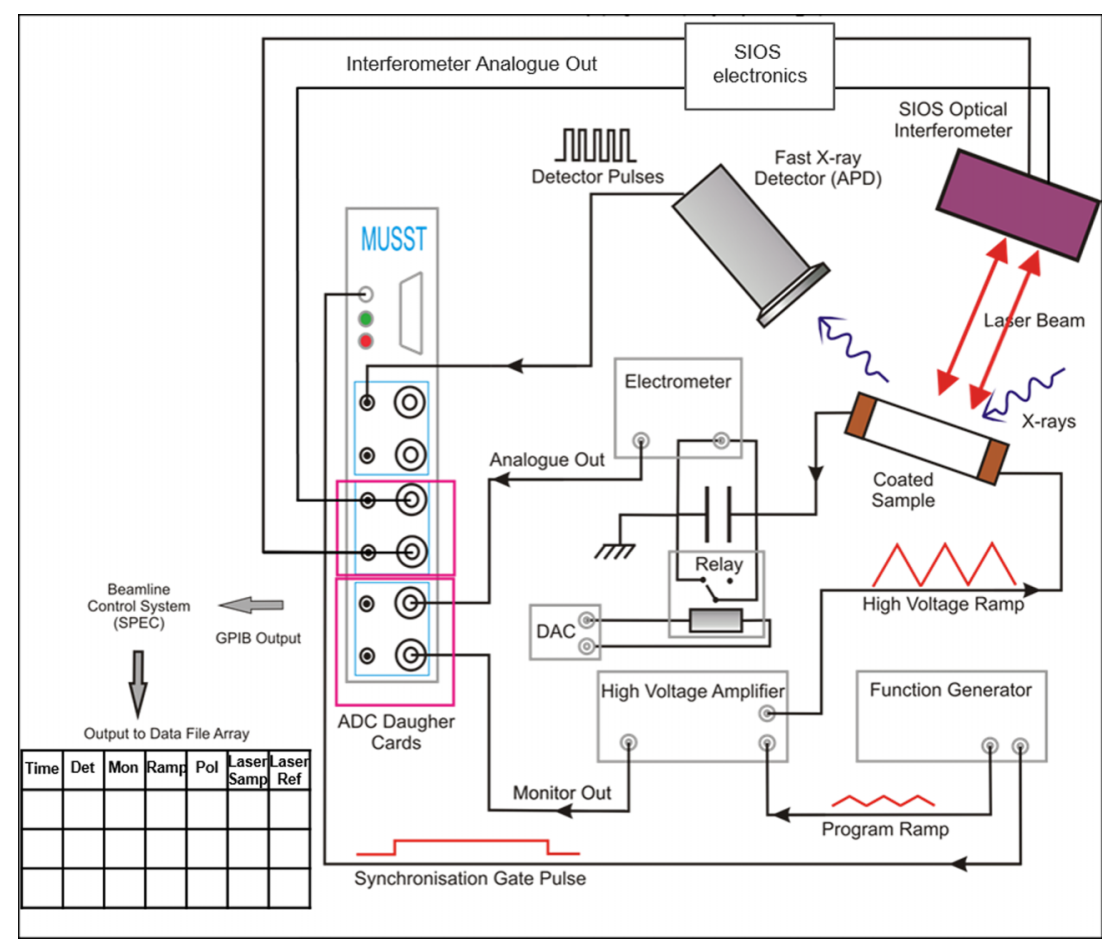

FIG. 3. The function generator is used to synchronize and control the experimental devices. The fast Avalanche Photo Diode (APD) X-ray point detector scans around the sample in $h k l$ space and collects diffracted X-rays. The data are recorded on the ultrafast digital-analogue converter MUSST card at the same time as the electrical data and interferometer data. 
TABLE I. Measurement range of the oscillation module SM-05 (Interferometer card): SIOS GmbH.

\begin{tabular}{lcc}
\hline \hline $\begin{array}{l}\text { Measurement } \\
\text { range no. }\end{array}$ & $\begin{array}{c}\text { Measuring range } \\
\text { factor in } \mu \mathrm{m} / \mathrm{V}\end{array}$ & $\begin{array}{c}\text { Maximum oscillation } \\
\text { amplitude in } \mu \mathrm{m}\end{array}$ \\
\hline 0 & 0.24 & 0.63 \\
1 & 0.97 & 2.50 \\
2 & 3.87 & 10.10 \\
3 & 15.48 & 40.30 \\
4 & 61.90 & 161.20 \\
5 & 247.61 & 644.90 \\
6 & 990.44 & 2579.50 \\
\hline \hline
\end{tabular}

from the two lasers in the interferometer. The data can be subsequently averaged and/or summed at $n$ points around the E-field cycle for improved counting statistics. When carrying out the experiment, $n$ should be at least equal or greater than 40 in order to obtain sufficient electric field resolution. This ensures that small variations of sample response as function of the applied electric field are tracked. Many multiple cycles (several thousands) of the E loop are usually necessary, especially for high frequencies, to collect sufficient integrated X-ray intensities. If the displacement is small, the same technique is applied to the interferometry data. The whole system is operated with the beamline control software, SPEC. ${ }^{24}$ The parameters of the MUSST card, the electric field (i.e., amplitude, frequency, waveform, number of cycles, and number of points to be acquired per cycle, amplitude offset, and phase: bipolar/unipolar cycles), and the interferometer (i.e., laser analogue gain as per Table I, alignment and offset corrections, and waiting times) are thus all configured before each acquisition. The acquisition and application of an AC electric field can be performed either within a scan (e.g., reciprocal lattice scan) or at a given fixed point in reciprocal space (to track relaxation, fatigue, or even sample degradation, beam damage, etc.). The way the system records and builds up the material response to a cyclic electrical field is presented in more detail in Ref. 20.

\section{E. Synchronization}

As shown in Figure 4, SPEC programming language macros have been written to set up all the hardware: once the macro is triggered by the user, the MUSST card is setup and starts to listen for a trigger signal to begin the acquisition. The macro sets up the function generator with the desired waveform, frequency, and number of cycles. In addition, the same macro sets up the interferometer by triggering the reset of the analogue output error (e.g., due to large movements of the diffractometer such as when moving from one reflection to a different one or signal drifts caused through mechanical relaxation of the diffractometer). It is also possible to trigger the reference mirror to reset the Lissajous figures between each diffractometer movement (e.g., after large movements). The Lissajous figure is a plot of one sinusoidal signal along the $\mathrm{x}$ axis (sample path) against another sinusoidal signal along the $\mathrm{y}$-axis (reference path). This method is used to determine the phase shift between the two sinusoidal signals and therefore to obtain a measure of distance/displacement. A global delay time can be set up. This is particularly important during a scan as it allows for the system to reach an equilibrium state (arising from motor damping and diffractometer relaxation) after a movement. Once the data acquisition is triggered, the macro sends the start signal to the function generator, which outputs a gate signal to the MUSST card (connected to the "sync" channel) to trigger the data acquisition, and a synchronous wave form (ramp) which drives the specimen. If a field larger than $10 \mathrm{~V}$ is required to drive the specimen, the ramp signal is
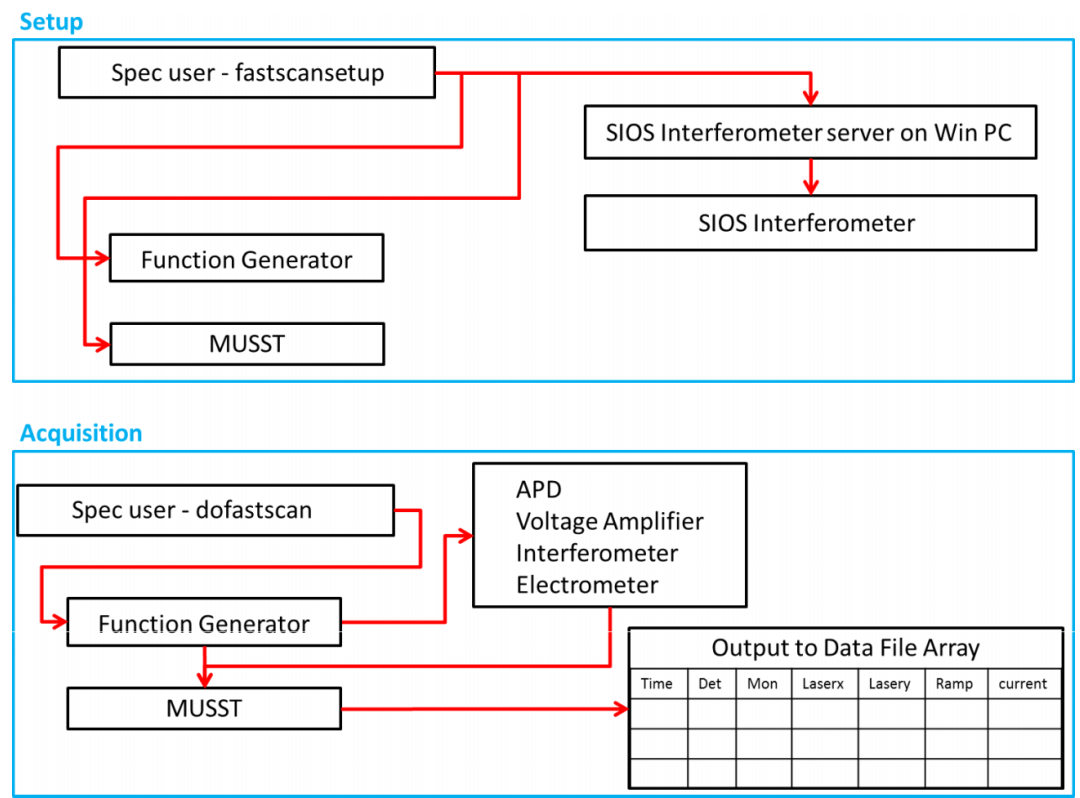

FIG. 4. (Top panel) Schematics of the software flow chart to setup the data acquisition: upon using the "start data acquisition" command and setting all the relevant parameters as explained in the text, the SPEC software sends the commands to the interferometer server, which sets up the interferometer, and to the function generator and MUSST card. (Bottom panel) Upon sending the "start data acquisition" command, the function generator is triggered and sends a gate signal to the MUSST card, which triggers all the other devices to start the acquisition. Once the acquisition is over, the data are sent to the PC and stored in a SPEC file as data file array. Additional information describing the parameters used to perform the acquisition is also stored in the headers of the same data file. 


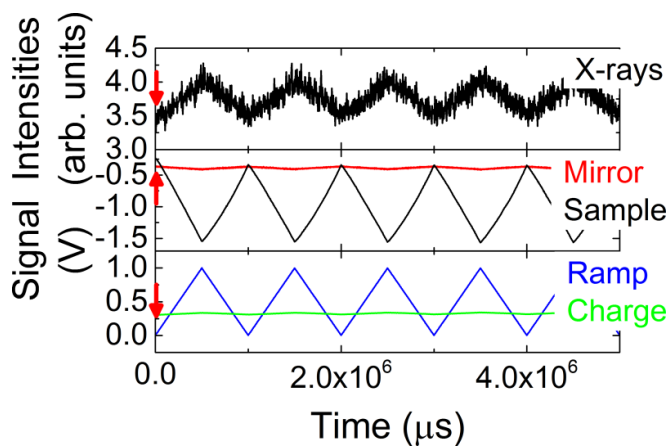

FIG. 5. (Top panel) X-ray intensities data collected as function of time in micro-seconds (AC field) in the reciprocal space position corresponding to the maxima of the (002) reflection. (Bottom panel) Ramp, measured charge and interferometry data (sample and reference mirror) as function of time in micro-seconds (AC field). The red arrow shows the simultaneous synchronization of the different signals thanks to the combination of MUSST card and function generator trigger.

connected to a TREK 610E high voltage amplifier. The X-ray photons measured with the APD detector, the polarization data (either from a Keithley 6514 electrometer connected to a Sawyer-Tower circuit, ${ }^{25}$ where the reference capacitor is selected via a capacitor bridge, or a current amplifier), and the two lasers (sample and reference), which are directly connected to the acquisition card, are simultaneously collected by the MUSST card at a maximum theoretical frequency of $\sim 1 \mathrm{kHz}$. The described system thanks to the MUSST card and the use of the function generator as a trigger ensures perfect synchronization of the data collected between the Xray diffraction patterns, the PE loop, and the interferometer data (Fig. 5).

\section{F. Data acquisition}

Polarization data are collected in situ,,$^{5,20,26}$ along with a measurement of global strain through the fringe and subfringe counting within a SIOS interferometer mounted above the sample as described in Sec. II E. X-ray data are collected in hkl-intensity format, where hkl represents the reciprocal space values in the crystallographic frame of reference. In order to determine the profile (position, intensity, and width) of a Bragg reflection of interest, rocking curves and hkl maps are typically measured. The electric field is cycled a large number of times (e.g., 1000) at each point in reciprocal space to build up counting statistics. The number of cycles required are dependent on the driving frequency chosen, the sample, and beamline setup. In the present case, due to the low frequency used and the large single crystal sample, only 5 cycles were necessary. The data are then post-processed to assign each part of the diffraction pattern to a particular electrical signal. Using fitting procedures, it is possible to extract the relevant Bragg peak parameters as function of AC field (peak position, intensities, and full-width-half-maximum). In this way, the three parameters (electrical polarization, global strain, and atomic strain) are presented as a function of applied electric field, Fig. 6. Note, the method adopted in this system assumes that the material properties do not change significantly over time (fatigue or aging for ferroelectric materials), but that the important materials features change with operational

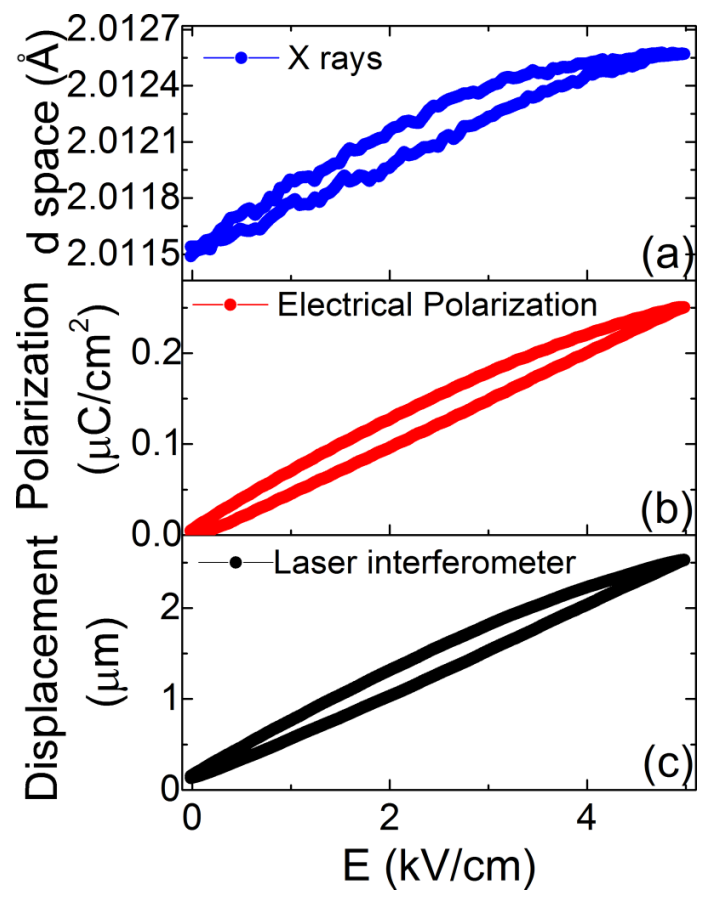

FIG. 6. Simultaneous determination of (a) lattice parameter, which is related to intrinsic strain contributions, (b) polarization, and (c) macroscopic displacement (related to intrinsic + extrinsic strain contributions) from a single crystal of PMT-PT as function of an unipolar ${ }^{28} \mathrm{AC}$ electric field up to $5 \mathrm{kV} / \mathrm{cm}$ (linear regime).

frequency. ${ }^{27}$ The continuous measurement of the charge or current with driving signal (if the PE loop measurement apparatus does not reset every cycle) does, however, allow an assessment of fatigue (permanent change in properties over cyclical excitation) for the piezoelectric or ferroelectric materials.

\section{G. Materials studied}

Samples were measured in a parallel plate capacitor geometry. For interferometry, a top mirror surface was required to reflect the laser beam. A gold top electrode (reflecting) a few hundred nanometer thick (to allow for wire bonding and low X-rays absorption) was evaporated. At least $250 \mu \mathrm{m}$ diameter contacts were used whenever appropriate. As large single crystal samples exhibit sizeable macroscopic strains ${ }^{2}$ (thus allowing reliable interferometry measurements), a $4 \times 4 \times 4 \mathrm{~mm}$ single crystal of lead magnesium niobate-lead titanate $\left((1-\mathrm{x}) \mathrm{Pb}\left(\mathrm{Mg}_{1 / 3} \mathrm{Nb}_{2 / 3}\right) \mathrm{O}_{3}-\mathrm{xPbTiO}\right.$ with $\mathrm{x}=0.32$, from TRS Ceramics, Inc., State College, PA) was chosen to assess the new experimental setup. The gold electrodes were evaporated on the [001] faces and poled in that orientation. The sample was then fixed on a glass substrate which had been previously gold coated. The coating was used both as electrode and as mirror surface for the reference beam.

\section{RESULTS AND DISCUSSION}

\section{A. Noise and metrology errors}

Vibration measurements were performed using vertical and horizontal L4-C geophones placed on the floor of the 
TABLE II. Peak to peak and rms (in brackets) displacements in $\mu \mathrm{m}$ measured on the XMaS diffractometer.

\begin{tabular}{llcccc}
\hline \hline Direction & Floor $(\mu \mathrm{m})$ & Upper stage $(\mu \mathrm{m})$ & Sample $(\mu \mathrm{m})$ & Detector $(\mu \mathrm{m})$ & $\begin{array}{c}\text { Sample-detector } \\
\text { relative motion }(\mu \mathrm{m})\end{array}$ \\
\hline$x$ & $0.89(0.1)$ & $0.97(0.11)$ & $1.02(0.12)$ & $1.30(0.17)$ & $0.61(0.09)$ \\
$y$ & $0.37(0.06)$ & $0.65(0.09)$ & $1.14(0.15)$ & $1.51(0.22)$ & $0.49(0.09)$ \\
$z$ & $1.35(0.13)$ & $1.43(0.14)$ & $1.41(0.14)$ & $1.41(0.14)$ & $0.21(0.02)$ \\
\hline \hline
\end{tabular}

beamline hutch, on the intermediate stage and at the sample and detector locations on the XMaS diffractometer.

The vibrational response of the structure to background excitation showed low frequency modes at 8 and $8.5 \mathrm{~Hz}$ in the horizontal directions (Table II). Some significant peaks are located at 10, 13.5, 17.6, and $21.6 \mathrm{~Hz}$. Some of these are related to excitation sources (not identified) present on the floor and are amplified by the diffractometer, in particular, $21.6 \mathrm{~Hz}$, while the first vertical mode is at $42.4 \mathrm{~Hz}$ with heavier damping. However, the relative motion between sample and detector is mainly affected by out of phase and de-correlated components and can reach $0.6 \mu \mathrm{m}$ and $0.2 \mu \mathrm{m}$ peak to peak in the horizontal and vertical directions, respectively. The low frequency contributions below $20 \mathrm{~Hz}$ have less impact. These are rigid body modes for which the structure is not deformed. The lack of rigidity of the floor/diffractometer interface is probably the reason for the observed low frequencies. At $21.6 \mathrm{~Hz}$ and up to $25 \mathrm{~Hz}$, the relative motion is significant in the horizontal directions. Since $21.6 \mathrm{~Hz}$ is also visible on the floor, it is assumed that it is caused by a vibration source nearby. However, since there is at least one mode of the diffractometer in that frequency region, the amplification with respect to the floor as well as the differential motion is significant. In the vertical direction, the measurement bandwidth was extended to $200 \mathrm{~Hz}$. The sample and detector responses are de-correlated above $100 \mathrm{~Hz}$.

Vibration measurements were also performed while various rotational motions were in progress. More particularly, the settling time of the mechanical structure when the rotation is stopped was investigated. Results show this settling time to be of the order of $200 \mathrm{~ms}$. Accelerometers were mounted at sample and detector positions measuring the vertical accelerations. The differential displacement between the two positions was computed using double time integration, see Table III.

Finally, the vibration modes were also measured directly using the interferometer and a $0.68 \mathrm{PMN}-0.32 \mathrm{PT}$ sample with no applied electric field. While at low frequencies $(<200 \mathrm{~Hz})$, the noise level is high and it reaches a flat background level of about $50 \mathrm{pm}$ at higher frequencies in static (no movement) conditions, as shown in Figure 7.

\section{B. Experimental challenges}

As shown in Figure 7, the resolution of the system is highly dependent on the operation frequency. In the case of thin films, where both the polarization and the displacement are very small, it is possible to modify the method to improve the measurement. A current amplifier could be used to directly measure the current, while a lock-in amplifier could be used for relatively high frequencies $(>10 \mathrm{~Hz})$ to increase signal to noise ratio and thereby separating the signal from the external vibrations. Such lock-in techniques would result in the loss of the dynamic displacement measurement capability as function of field. However, preliminary tests without a lockin amplifier have shown that if enough data are collected, $\mathrm{nm}$ displacement measurements are possible. The described procedures are relatively straightforward in the case of large single crystal samples (assuming the sample/reference surface angle is smaller than $1^{\circ}$ which could be corrected by the use of the wedge focusing lenses). For thin films, however, it is much more challenging. One issue is the size of the electrodes, which, in films, could be of few $\mu$ m or smaller. The convolution between the diffractometer sphere of confusion, the laser alignment with the center of rotation, and the sample electrode could lead to a precession of the beam on the sample surface and a possible underestimation of the measured displacement (though improved methods exist such as the "edge" of the electrode technique ${ }^{29}$ or use of back reflections from the mirror reference in case of a transparent sample). Additionally, another source of misalignment is the change of shape of the sample itself due to the external applied stimuli. For single crystals and thin films, if the initial alignment was perfect (reflected laser hitting exactly the center of the optics), this effect is minimized by the angular acceptance of the focusing lenses. Thereby in the system described in this paper, the laser would need to move by $\sim 1.5 \mathrm{~mm}$ to exit the focusing lenses.

TABLE III. Peak to peak displacements in $\mu$ measured on the XMaS diffractometer estimated from acceleration data (numerical double time integration and high pass filtering). Settling times are approximate values of the time taken to go back to background level when motion has stopped.

\begin{tabular}{lcccc}
\hline \hline Rotation $(\mu \mathrm{m})$ & Sample $(\mu \mathrm{m})$ & Detector $(\mu \mathrm{m})$ & Sample-detector difference $(\mu \mathrm{m})$ & Settling time $(s)$ \\
\hline Background & 0.36 & 0.39 & 0.05 & $\ldots$ \\
$\theta$ & 1.56 & 1.61 & 0.46 & 0.2 \\
$2 \theta$ & 0.47 & 0.53 & 0.08 & 0.2 \\
$\chi$ & 1.51 & 1.98 & 1.90 & 0.2 \\
$\phi$ & 0.41 & 0.51 & 0.20 & 0.1 \\
\hline
\end{tabular}




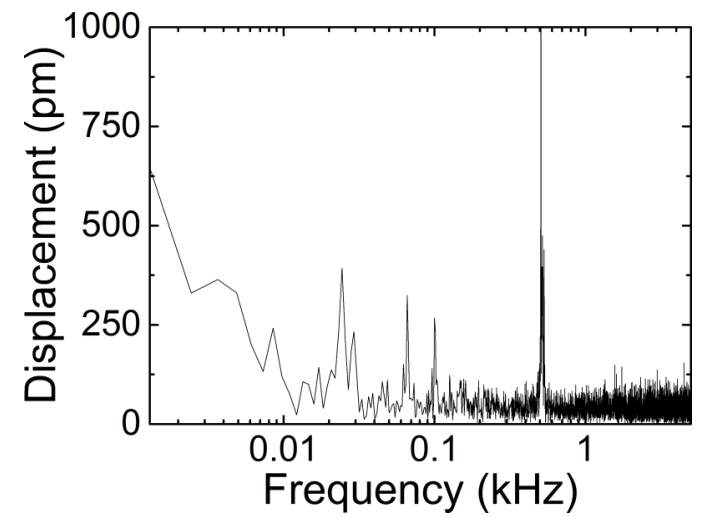

FIG. 7. Frequency dependent vibrations as measured with the laser interferometer using a single crystal $0.68 \mathrm{PMN}-0.32 \mathrm{PT}$ sample mounted on a gold coated glass window acting as reference surface. The plot shows the difference between the sample and reference displacement. The frequency is presented as a log scale.

\section{Experimental results}

In order to transform the raw data measured in volts to displacement expressed in micrometers, data reduction procedures have been written in a custom designed data analysis code. Details of these procedures are as follows: The reference surface (mirror) displacement is subtracted from the sample displacement and then the data are averaged in a single loop as shown in Figure 6(c). The averaged data are then corrected by the amplification factor as per Table I for the $\mathrm{V}$ to $\mu \mathrm{m}$ conversion. A similar procedure is performed for the X-ray (Fig. 6(a)) and the polarization datasets (Fig. 6(b)): the APD data are normalized to the X-rays beam monitor and corrected for the acquisition time and beam attenuation; the polarization data are extracted from the charge by dividing by the electrode area or by integration of the current and area normalization, depending on the setup (Sawyer-Tower circuit or current measurements).

The system response has been tested as function of electric field amplitude, with particular attention to the low voltage regime $(\sim 10 \mathrm{~V})$, which corresponds to the crossover region between the TREK high voltage amplifier and the function generator. As shown in Figure 8, it has been found that the TREK data appear to be shifted by an offset voltage value of $0.012 \mathrm{~V}(\mathrm{E}=0.0302 \mathrm{kV} / \mathrm{cm})$, value determined by measuring the voltage output of the amplifier at $V=0$ input. This small offset could be disregarded in most cases during an experiment, although it needs to be taken into account whenever the data are compared with data acquired from other labs - a comparison made easier by this measuring system by the simultaneous acquisition of the electrical response (PE) data.

As shown in Figure 1, the laser interferometer measures the displacement component along the vertical direction $\left(\Delta \mathrm{L}_{\text {Sample }}=\Delta \mathrm{L}-\Delta \mathrm{L}_{\text {reference }}\right)$, which in the present crystal geometry corresponds roughly to the [001] direction, which is also the direction of poling. Different modes of operation of the diffractometer are available. For this experiment, we used a "fixed $\phi$ " mode ${ }^{24}$ (fixed rotations along the [001] axis) in order to minimize the $\phi$ rotations, which are decoupled from the interferometer. In extreme cases, the $\phi$ angle can

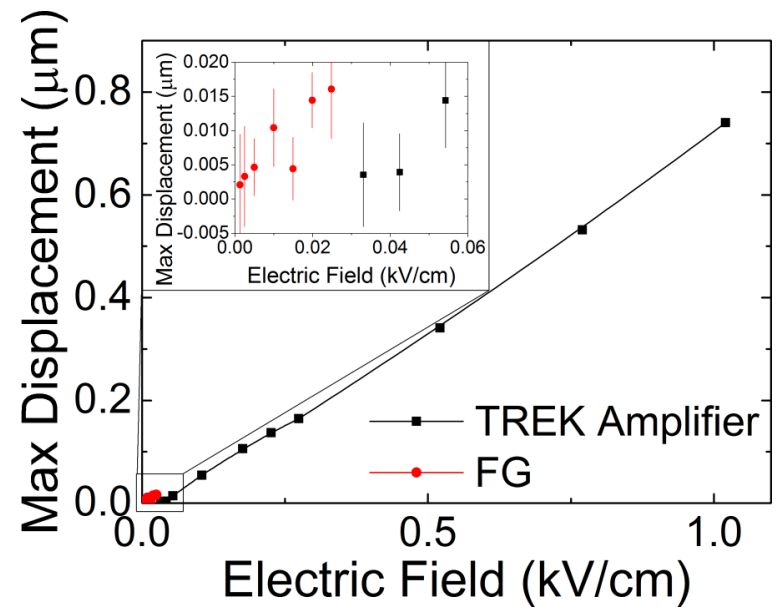

FIG. 8. Maximum displacement at $10 \mathrm{~Hz}$ as measured with the TREK high voltage amplifier and the function generator as low power supply. The line is a guide to the eye. The inset shows details of the crossover region at low electric fields between the two setups.

be used to align on a particular reflection and to "close" the scattering triangle. However, a realignment may then be necessary depending on the sample/mirror surfaces angle. To minimize this effect, an optimal $\phi$ angle should be set in order to allow the maximum coverage of the desired reflections. In the present experimental case (incident photon energy of $11.8 \mathrm{keV}$, just below the gold $\mathrm{L}_{3}$ edge to minimize fluorescence from the electrodes), the $\phi$ angle was chosen in order to have access to the [002], [022], and [222] reflections without further $\phi$ movements. While the specular [002] reflection is directly proportional to the $d_{33}$ piezoelectric coefficient and parallel to the measured laser displacement, the [022] and [222] reflections are related to a convolution between the $\mathrm{d}_{33}, \mathrm{~d}_{31}$, and $\mathrm{d}_{15}$ coefficients. For convenience, the data shown in Figure 6 refer to the [002] Bragg reflection, which can be compared directly to the interferometry data. The lower statistics for the $\mathrm{X}$-ray data, when compared to both the laser interferometry and the electric polarization data, is shown in Figure 6. In order to improve the noise/data ratio, it is possible to count for longer (e.g., larger number of electric field cycles) and/or rebin the data in fewer electric field/reciprocal space points. Due to the large $d_{33}$ shown by the investigated sample, no crystal analyser has been used, however, for samples showing small $\mathrm{d}_{33}$ values, and the use of a crystal analyser for improved $\mathrm{X}$-rays resolution should be considered.

In Figure 9, the intrinsic (X-rays) and intrinsic + extrinsic (laser) strain are shown together on the same scale. The lattice parameter strain is obtained from measuring the position of the diffraction peaks in angular space at zero and under an applied electric field. These positions, which are related to the lattice parameters, can be used to calculate the strain by

$$
\epsilon_{h k l}=\left(d_{h k l}^{E}-d_{h k l}^{0}\right) / d_{h k l}^{0},
$$

where $d_{h k l}^{E}$ is the $d$-spacing for a given (hkl) reflection under applied electric field and $d_{h k l}^{0}$ is the $d$-spacing for the same reflection with zero applied electric field.

The displacement measured by the interferometer is the sample minus the mirror displacement at a given electric field, with $\mathrm{E}=0$ set as zero displacement. The simultaneously 


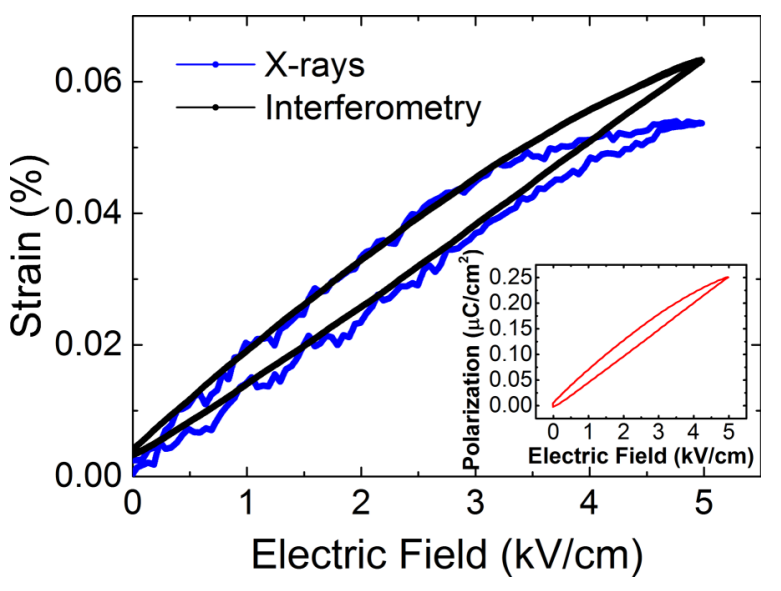

FIG. 9. Strain dependence as function of applied ac (frequency $=1 \mathrm{~Hz}$ ) electric field of a 0.68PMN-0.32PT single crystal on the [002] specular reflection. Blue line shows the lattice (from x-ray data) intrinsic contribution and the black line represents the strain as measured by the laser interferometer. Inset: the red line represents the simultaneously measured electric polarization for an electric field from 0 to $5 \mathrm{kV} / \mathrm{cm}$.

measured electric polarization is shown in the inset. The selected field is approximately within the linear range of the material and therefore neither jump in polarization or butterfly shape strain loops are expected nor are visible. Note though that in these highly electrostrictive materials, one would not expect as high a degree of hysteresis as found in conventional ferroelectric piezoceramics—such as PZT, for example. Figure 9 shows the dependence of intrinsic and extrinsic strains as function of electric field at $1 \mathrm{~Hz}$. Here, at low fields, little to no extrinsic contribution is present, while at about $3 \mathrm{kV} / \mathrm{cm}$, extrinsic effects often associated with domain wall motion start to play an important role in the total piezoelectric behavior of the measured 0.68PMN$0.32 \mathrm{PT}$ single crystal, and the trends between X-ray and interferometry data start to be different. This is the expected behavior in this class of material.

\section{CONCLUSIONS}

In this paper, we have described the new capability that allows simultaneous electrical and mechanical characterizations of the functional properties of ferroelectric materials to be recorded together with detailed and time-synchronized crystallographic information. The time-synchronization equates directly to electric field synchronization in the system developed. The global strain as measured by the laser interferometer can be directly related to the atomic strain probed by high resolution synchrotron $\mathrm{X}$-ray diffraction. The aim of this work is to enable the understanding of the atomic and microstructural evolution of the material properties whilst simultaneously measuring the overall strain (and electrical) characteristics. The combined information (Fig. 9), which links together strain at several lengths scales from nm (interferometry) to fm (diffraction) as well as time scales from $0.01 \mathrm{~Hz}$ to presently $55 \mathrm{~Hz}$, allows new insights to be gleaned on the correlation between induced strain and material properties-information of crucial importance for the development of novel devices such as IBM's patented piezoelectric effect transistor. ${ }^{17,30}$
Since the origin of the extraordinarily large electromechanical coupling and piezoelectric coefficients within 0.68PMN$0.32 \mathrm{PT}$ at the morphotropic phase boundary is not yet adequately explained in the literature, the ability to tune the piezoelectric response through domain engineering (with different orientations of crystal cuts, poling and/or direction of working electrodes) offers a variety of geometries that may be investigated within the novel experimental development on the XMaS beamline. The incident angle and the energy tunability that a synchrotron beamline offers allow the investigation of samples as function of $\mathrm{x}$-ray penetration depth together with elemental sensitivity/selectivity. The measurement capability described here, which has been developed for ferroelectric materials characterization, has great potential to be used in different scientific areas such as electrochemistry, magnetism (magnetostriction and multiferroics), and others. Further developments currently underway include a temperature stage, an AC magnetic field capability, and high frequency investigations up to the $\mathrm{MHz}$ regime, which will form the basis of future publications.

\section{ACKNOWLEDGMENTS}

This work was funded through the European Metrology Research Programme (EMRP) Project IND54 Nanostrain. The EMRP is jointly funded by the EMRP participating countries within EURAMET and the European Union. Funding was received from the UK's National Measurement System for development of the metrology of the PE loop and integration within the beamline. ${ }^{31}$ This work was performed on the EPSRC-funded XMaS beamline at the ESRF, we are grateful to S. Petitdemange for software support. We also thank Rob Roach and Peter Grundschok for their support with the interferometer system and Scanwel ${ }^{32}$ for manufacturing the specialized sample holder.

${ }^{1}$ M. Stewart, M. G. Cain, and D. Hall, "Ferroelectric hysteresis measurement and analysis," NPL Report CMMT(A)152, 1999.

${ }^{2}$ S.-E. Park and T. R. Shrout, J. Appl. Phys. 82, 1804 (1997).

${ }^{3}$ K. T. Jacob and L. Rannesh, Mater. Sci. Eng., B 140, 53 (2007).

${ }^{4}$ M. Davis, J. Electroceram. 19, 25 (2007).

${ }^{5}$ J. Wooldridge, M. Stewart, C. Vecchini, M. G. Cain, M. Gutmann, and M. Reece, J. Am. Ceram. Soc. 97, 2111 (2014).

${ }^{6}$ D. Damjanovic and M. Demartin, J. Phys.: Condens. Matter 9, 4943 (1997).

${ }^{7}$ D. Damjanovic and D. Damjanovic, IEEE Trans. Ultrason., Ferroelectrics, Freq. Control 56, 1574 (2009).

${ }^{8}$ D. Damjanovic, J. Am. Ceram. Soc. 88, 2663 (2005).

${ }^{9}$ D. Damjanovic and M. Demartin, J. Phys. D: Appl. Phys. 29, 2057 (1996).

${ }^{10}$ D. Damjanovic, Phys. Rev. B 55, R649 (1997).

${ }^{11}$ Z. Ye and M. Dong, J. Appl. Phys. 87, 2312 (2000).

${ }^{12}$ M. Abes, C. T. Koops, S. B. Hrkac, E. Quandt, L. Bouchenoire, B. M. Murphy, and O. M. Magnussen, J. Appl. Phys. 113, 124303 (2013).

${ }^{13}$ M. Davis, D. Damjanovic, and N. Setter, Phys. Rev. B 73, 014115 (2006).

${ }^{14}$ K. Chen, X. Zhang, and H. Luo, J. Phys.: Condens. Matter 14, L571 (2002).

${ }^{15}$ X. Zhao, B. Fang, H. Cao, Y. Guo, and H. Luo, Mater. Sci. Eng., B 96, 254 (2002).

${ }^{16}$ E. A. McLaughlin, T. Liu, and C. S. Lynch, Acta Mater. 52, 3849 (2004).

${ }^{17}$ D. M. Newns, B. G. Elmegreen, X. H. Liu, and G. J. Martyna, MRS Bull. 37, 1071 (2012).

${ }^{18}$ D. Paul, M. Cooper, and W. Stirling, Rev. Sci. Instrum. 66, 1741 (1995).

${ }^{19}$ S. Brown, L. Bouchenoire, D. Bowyer, J. Kervin, D. Laundy, M. Longfield, D. Mannix, D. Paul, A. Stunault, P. Thompson, M. Cooper, C. Lucas, and W. Stirling, J. Synchrotron Radiat. 8, 1172 (2001).

${ }^{20}$ J. Wooldridge, S. Ryding, S. Brown, T. L. Burnett, M. G. Cain, R. Cernik, R. Hino, M. Stewart, and P. Thompson, J. Synchrotron Radiat. 19, 710 (2012). 
${ }^{21}$ See www.sios.de for Sios meßtechnik gmbh.

${ }^{22}$ U. Kogelschatz, B. Eliasson, and M. Hirth, Ozone Sci. Eng. 10, 367 (1988)

${ }^{23}$ C. Weilandics, N. Rohrig, and N. F. Gmur, "Ozone production at the national synchrotron light source," Technical Report No. BNL-39351 (National Synchrotron Light Source, Brookhaven National Laboratory, 1987).

${ }^{24}$ See www.certif.com/ for Certified scientific software spec.

${ }^{25}$ C. Sawyer and C. Tower, Phys. Rev. 35, 269 (1930).

${ }^{26}$ S. Ryding, R. Cernik, J. Wooldridge, T. L. Burnett, M. Stewart, C. Vecchini, M. G. Cain, A. Lennie, F. Yuan, C. Tang, and P. Thompson, Powder Diffr 28, S220 (2013).
${ }^{27}$ Polar Oxides Properties, Characterization, and Imaging, edited by R. Waser, U. Bottger, and S. Tiedke (Wiley-VCH Verlag GmbH, 2005).

${ }^{28}$ F. Fang, W. Yang, F. C. Zhang, and H. Qing, J. Mater. Res. 23, 3387 (2008). ${ }^{29}$ M. Stewart, S. Lepadatu, L. N. McCartney, M. G. Cain, L. Wright, J. Crain, D. M. Newns, and G. J. Martyna, APL Mater. 3, 026103 (2015).

${ }^{30}$ D. Newns, B. Elmegreen, X. Hu Liu, and G. Martyna, J. Appl. Phys. 111, 084509 (2012).

${ }^{31}$ Characterisation of Ferroelectric Bulk Materials and Thin Films, edited by M. G. Cain (Springer, Netherlands, 2014).

${ }^{32}$ See www.scanwel.co.uk for Scanwel Ltd. 